\title{
INTERNET, REDES SOCIALES Y ADOLESCENCIA: UN ESTUDIO EN CENTROS DE EDUCACIÓN SECUNDARIA DE LA REGIÓN DE MURCIA ${ }^{1}$
}

\section{THE INTERNET, SOCIAL NETWORKS AND ADOLESCENCE: A STUDY BASED ON SECONDARY SCHOOLS OF THE REGION OF MURCIA}

\author{
F. Javier Ballesta Pagán \\ Josefina Lozano Martínez \\ $M^{a}$ Carmen Cerezo Máiquez \\ Universidad de Murcia \\ Encarnación Soriano Ayala \\ Universidad de Almería
}

Fecha de recepción: 15/10/2014

Fecha de aceptación: 04/12/2014

\section{RESUMEN}

Con este artículo pretendemos dar a conocer la influencia de las redes sociales en la relaciones que establecen los jóvenes con otros a través de este medio; al mismo tiempo, conocer el control ejercido por las familias sobre el uso de las redes sociales en la vida diaria de los adolescente de algunos centros de la Comunidad Autónoma de la Región de Murcia.

El grupo investigador ha pretendido analizar, con un interés particular, las relaciones sociales y el control que ejerce el ámbito familiar ante el uso de las redes sociales del alumnado de Educación Secundaria Obligatoria. De acuerdo con nuestro objetivo general de investigación, optamos por una metodología cuantitativa, utilizando la encuesta método y para este propósito se diseñó un cuestionario compuesto por 67 ítems en la escala Likert, distribuidos en 11 dimensiones. Debido a la amplitud de la investigación, en este estudio sólo se presentan los resultados obtenidos en relación con la dimensión "Tu tiempo libre", centrándonos en el uso de las TIC, como medio de ocio.

De los resultados obtenidos se constata que la mayoría considera que Internet es un lugar seguro y que nunca han sido acosados a través de las redes sociales, al mismo tiempo que toman medidas de seguridad al "subir" sus fotos, contactar con desconocidos o han tenido una cita con personas que no conocen. Así pues, los jóvenes no ven un uso inadecuado de las redes sociales y piensan que la influencia en sus vidas no les perjudica.

Palabras clave: Redes sociales, alumnado autóctono, alumnado extranjero, control de las familias, Internet.

\footnotetext{
${ }^{1}$ Estos datos son del Proyecto Nacional Educación I+D Ministerio de Educación (EDU2011-26887) "Educación para la salud transcultural en los jóvenes, inmigrantes y autóctonos de Almería: análisis e intervención para su optimización y mejora".
} 
Internet, redes sociales y adolescencia: un estudio en centros de Educación Secundaria de la región de Murcia

\begin{abstract}
With this article we intend to publicize the influence of social networks in the relations established with other young people through this medium; while knowing that exerted by families on the use of social networks in the daily life of teenager some centers of the Autonomous Community of the Region of Murcia control.

The research group has tried to analyze, with a particular interest, social relations and the control exercised by the family to the use of social networks of students of Secondary Education. According to our overall goal of research, we opted for a quantitative methodology, using the survey method and for this purpose a questionnaire consisting of 67 items in the Likert scale, distributed in 11 dimensions was designed. Because of the breadth of research, this study only the results obtained in relation to the dimension "Your time", focusing on the use of ICT as a means of entertainment are presented.

From the results it appears that the majority believes that the Internet is a safe place and never have been harassed through social networks, while taking security measures to upload their photos, contact with strangers or had an appointment with people who do not know. So, young people do not see an inappropriate use of social networks and think the influence in their lives does not harm them.
\end{abstract}

Key words: Social networking, native students, foreign students, families control, Internet.

\title{
1. INTRODUCCIÓN
}

Las Tecnologías de la Información y de la Comunicación (TIC) forman parte, cada vez más, de nuestras vidas y están presentes en nuestros hogares y en las aulas escolares. Estamos ante un fenómeno de creciente evolución que se ha visto incrementado en las últimas décadas a una velocidad vertiginosa, constituyéndose como uno de los fenómenos más característicos del escenario social actual. Sabemos que los jóvenes utilizan los medios digitales para el establecimiento de relaciones sociales y para ocupar su tiempo de ocio (Buckingham, 2008; Bringué \& Sádaba, 2009; Rubio, 2009, Sánchez \&Fernández, 2010) destacan que sobre el acceso y uso de medios digitales por parte de los jóvenes, subrayan la necesidad de profundizar sobre la relación con el contexto familiar y sociocultural, con la finalidad de favorecer una educación en y para el uso y consumo de medios digitales Además, suelen hacer uso de las TIC de manera individual y dentro del hogar (Buckingham, 2007, 2008).

El informe Sociedad de la Información (2013) menciona que el acceso al mundo cibernético ha evolucionado de tal forma que, aproximadamente, el $23 \%$ de las personas se conectan a través de una televisión, $63 \%$ a través de un ordenador, seguido de $74 \%$ que utilizan una portátil, el $81 \%$ utiliza las tabletas y, por último, el $83 \%$ lo hace a través de los teléfonos móviles. Para González-Jiménez (2013) y Romero 
\& Díez, (2013) este aumento en el acceso a Internet a través de un teléfono móvil está causando cambios en el comportamiento y en la mentalidad de los ciudadanos, ya sea por las compras que hacen (de acuerdo con este estudio, el $25 \%$ de los usuarios hacen compras utilizando teléfonos móviles) o en el campo de las relaciones sociales para hacer amigos o citas. Principalmente este acceso a Internet se lleva a cabo en esas tareas llamados micro-actividades, que se produce cuando tenemos tiempo "muerto" en nuestras manos, que usamos para acceder a Internet, por ejemplo, cuando estamos en línea esperando para pagar en una tienda, esperando a un amigo, etc. Según Bernal \& Angulo (2012) el uso y acceso de las redes sociales ha llegado a ser un nuevo entorno de socialización para los jóvenes, espacio para la construcción de la identidad social con sus iguales, a veces con ningún control o asesoramiento parental; aún conociéndose que la inmersión en las mismas es cada vez más prematuro y por debajo de la edad mínima permitida. La mayoría de los alumnos utilizan las redes sociales principalmente para divertirse y ponerse en contacto con sus amigos, también para conocer otros e intercambiar mensajes e imágenes, una actividad que realizan desde hace tiempo y que reconocen no quitar tiempo a otras actividades (Ballesta, Lozano \& Cerezo, 2014).

Por consiguiente y desde este planteamiento cabría profundizar sobre algunas cuestiones referidas a ¿̇qué uso hacen los adolescentes de Internet y cómo navegan por las redes sociales?, ¿los jóvenes son asesorados por sus familias para conocer los riesgos que ello puede tener?, ¿están formados para vivir una realidad digital con sus ventajas y sus limitaciones? Para dar respuestas a estos interrogantes nos remitimos a aquel estudio realizado por el Observatorio del Instituto Nacional de las Tecnologías de la Información (2010) titulado "Estudio sobre seguridad y privacidad en el uso de la telefonía móvil en España ", muestra que 8 de cada 10 adolescentes, entre 15 y 16 años de edad son dueños de su propio teléfono móvil personal. Ellos los usan para tomar imágenes (88\%), que se publican en Internet $(20,8 \%)$ y se envía a otras personas $(48,2 \%)$. Si echamos un vistazo a un grupo de edad más joven, los datos de esta investigación indican que 2 de cada 3 personas de entre 10 y 16 años poseen un teléfono móvil. Cerca del $4 \%$ de ellas confirman que tiene fotos 0 videos de carácter provocativo y $8,1 \%$ afirman que han recibido información de extraños. Estos datos muestran que los menores de edad, así como los adolescentes están formando parte de una realidad digital y en consecuencia, se requerirá una formación específica en este sentido.

Una de las consecuencias de un uso inadecuado de los teléfonos móviles por los menores y adolescentes es la práctica llamado sexting 
Internet, redes sociales y adolescencia: un estudio en centros de Educación Secundaria de la región de Murcia

que consiste en el envío de fotos y/o videos de carácter sexual a otras personas mediante dispositivo móvil teléfono. Esta información es creada principalmente por el remitente (Unicef, 2012). De esta práctica, una serie de consecuencias surgen para el remitente como ser objeto de chantaje, la humillación pública, la falta de respeto a su honor, la integridad y la imagen, la pornografía infantil y ciberbullying, entre otros. Pensemos que una de las características destacables en el uso de las redes sociales es que los usuarios pueden optar por el anonimato o asumir identidades falsas, mientras que ellos aceptan y hablan con otros usuarios, incluidos los menores de edad. A partir de esta base, nos preguntamos cómo podemos proteger a nuestros menores contra el uso de tecnologías de la información como las redes sociales o un uso inadecuado de un teléfono móvil.

En este sentido, Durán \& Guerra (2015) afirman que las redes sociales, tipo Facebook, MySpace, Bebo o Tuenti, permiten a sus usuarios desarrollar espacios compartidos, visibles para sus "amigos 0 contactos" en los que es posible crear perfiles públicos individuales, e interactuar tanto con amigos con los que se mantiene un contacto fuera de la red como con personas con las que no se mantiene una relación más allá de la propia red social. Así, como apunta Becoña (2006), las redes sociales pueden "atrapar" a las personas, sobre todo adolescentes y jóvenes, ya que el mundo virtual contribuye a crear una falsa identidad y a distanciarlas o a distorsionar su mundo real, generando de esta manera un entorno propicio para el desarrollo de una conducta adictiva. Por otra parte, como afirman Tejedor \& Pulido (2012) el acoso entre iguales mediado por las interacciones on-line, genera un impacto muy negativo en su desarrollo emocional; depresiones, baja autoestima, y en el último extremo el suicidio, además señalan la importancia de promover una formación crítica y prevención no solo limitada a los y las menores, sino ampliada a toda la comunidad.

Por otra parte, el estudio de las redes sociales utilizadas por jóvenes es especialmente relevante en tanto que ellos priorizan estas formas de comunicación respecto a las tradicionales, basadas en el contacto personal directo. Como señalan Colás, González \& De Pablos (2013) algunas investigaciones recientes muestran que la comunicación social on-line de jóvenes está influida por la percepción de su identidad y autoestima, así como la compensación social y entorno social. Por eso sugieren que las redes sociales on-line son para la juventud fuente de recursos que son utilizados para cubrir necesidades, tanto de índole psicológica como social. Sin embargo, las diferencias entre sexos en estas variables demuestra que tienen un papel compensatorio, ya que son los hombres los que mayormente recurren a ellas para cubrir facetas 
emocionales y reforzar su autoestima, mientras en el caso de las jóvenes prima una función relacional.

\section{USO DE LAS REDES SOCIALES POR EL ADOLESCENTE Y REGULACIÓN FAMILIAR}

Como estamos comprobando los jóvenes, cada vez con mayor frecuencia, se expresan, preferentemente, a través de sistemas de comunicación virtual y la utilización de las redes sociales se hace cada vez más extensiva (Becoña, 2006). Al mismo tiempo, nos planteamos la influencia que puede tener la familia en este nuevo hábito por parte de sus hijos adolescente y cómo puede modular y regular su consumo. Incluso, por llegar más lejos, sería bueno saber si son las familias de estos adolescentes conocedoras del uso que hacen sus hijos de dichas redes sociales.

Para O'Keeffe \& Clarke-Pearson (2011) la familia puede ser un agente importante para la regulación de los usos de Internet y las redes sociales en los menores, aunque se ha pasado de mostrar cierto temor, por parte de los padres, en contraste con los adolescentes que no veían ninguna preocupación al respecto (Livingstone, 2008) a un actitud mucho más permisiva y desigual evidenciando, a la vez, discrepancias en cuanto a la percepción de riesgo por parte de algunos padres e hijos, y cómo estos últimos no intuyen peligro en algunas prácticas de riesgo tales como contactar con desconocidos, mostrar datos privados o hacer una utilización predominante de la red para el manejo de sus relaciones sociales (McCarty, Prawitz, Derscheid \& Montgomery, 2011). Como se puede encontrar en el estudio realizado por Rial, Gómez, Braña \& Varela (2014) el $36.2 \%$ de los adolescentes manifiesta no conectarse todo lo que le gustaría a Internet, pero tan solo en el $46 \%$ de los casos existe un control parental al respecto. Estaríamos hablando de un $52.8 \%$ de escolares a los que sus padres no controlan, ni ponen límite alguno a la hora de usar Internet. Un dato complementario es que el $77.9 \%$ de los chicos/as que utilizan Internet, no han tenido nunca o casi nunca una discusión con sus padres por su uso; mientras que el $6.3 \%$ manifiesta tener problemas muchas veces y el 14.6\%, de vez en cuando.

El proyecto europeo EU Kids Online (Livingstone \& Haddon, 2011), elaborado al amparo de la Comisión Europea, evidencia que con frecuencia los padres no son conscientes de los riesgos que experimentan sus hijos en Internet: solo un $28 \%$ de los padres bloquea o filtra webs y únicamente un $23 \%$ realiza un seguimiento de las webs visitadas por sus hijos. 
Internet, redes sociales y adolescencia: un estudio en centros de Educación Secundaria de la región de Murcia

Ante la influencia que los medios digitales están protagonizando en los jóvenes, la educación no puede mirar para otro lado. En este sentido, puede ser significativo aproximarse y conocer las experiencias que los jóvenes desarrollan con dichos medios, es decir, es importante dar respuesta a las siguientes cuestiones: żqué uso y consumo realizan los jóvenes de ciertos centros escolares de Educación Secundaria en la Región de Murcia (España) en relación a Internet y en concreto a las redes sociales?, ¿̇es posible que estas nuevas formas de uso y consumo estén originando cambios en las formas de pensar, sentir y actuar de dicho sector de la población?, żlas familias de estos adolescentes son conocedoras del uso que hacen sus hijos de las redes sociales?, ¿̇saben si sus hijos han sido acosados a través de ellas? i los jóvenes, en caso de acoso a través de las redes sociales, lo comunican a sus padres?.

\section{METODOLOGÍA DE LA INVESTIGACIÓN}

\subsection{Diseño}

Nuestra investigación se encuadra dentro de una metodología de encuesta que intenta obtener información cuantitativa sobre una población en términos descriptivos. Se ha utilizado un diseño transversal en consonancia con la finalidad investigadora y como instrumento de recogida de información, la encuesta. De este modo, se ha recogido información de una muestra grande como la nuestra al poder ser aplicado simultáneamente y en poco tiempo a un número elevado de alumnos de Educación Secundaria Obligatoria, así como posibilitaba obtener una información referida a aspectos muy diversos relacionados con los objetivos planteados.

\subsection{Instrumento}

Para la recogida de información se utilizó un cuestionario conformado por 67 ítems con cinco opciones de respuesta y estructurados en once dimensiones: datos de identificación, salud, alimentación y actividad física, sentimientos, estados de ánimo, sobre ti..., tu tiempo libre, tu vida familiar, tus amigos, el instituto/colegio, tú y los demás, más sobre ti...

A través de los datos de identificación pretendíamos recoger información sociodemográfica de interés sobre el alumnado participante y su familia (sexo, edad, país de nacimiento del alumno, del padre y de la madre, enfermedad o problema médico crónico y nivel de estudios). 
Debido a la amplitud de la investigación, en este estudio sólo se presentan los resultados obtenidos en relación con la temática Tu tiempo libre, centrándonos en el uso de las Tecnologías de la Información y de la Comunicación (TIC) como medio de ocio, relación y entretenimiento.

El instrumento tiene una fiabilidad, Alfa de Cronbach de 0,72; considerada según Bisquerra $(1989,2012)$, como media o alta, lo que nos indica que el instrumento tiene una media-alta coherencia interna.

\subsection{Objetivos}

- Conocer la influencia y el riesgo de las redes sociales en las relaciones de los adolescentes.

- Valorar el papel de las familias sobre el uso de sus hijos de Internet.

\subsection{Participantes}

En esta investigación han participado 119 alumnos escolarizados, durante el curso 2013-2014, de Educación Secundaria Obligatoria en 5 centros docentes públicos y concertados de la Región de Murcia, que venían desarrollando Proyectos en Educación para la Salud. Del total de alumnos, $19.3 \%$ son de origen extranjero y el $80.7 \%$ son de origen autóctono.

\subsection{Procedimiento}

Tras el proceso de selección de centros y alumnado, el equipo investigador se puso en contacto con los directores de los centros, y se les propuso que sus alumnos implementaran el cuestionario vía on-line, para ello se utilizó una de las sesiones de tutoría, previo acuerdo con los orientadores de los centros educativos.

Una vez recogidos los datos fueron analizados con el paquete estadístico SPSS for Windows 19.0, utilizando estadísticos descriptivos (frecuencias) y estadísticos inferenciales, para realizar cruces entre diferentes variables comprobando las relaciones de dependencia que se pueden establecer entre diferentes variables que hipotéticamente pueden resultar estadísticamente significativas, obviando aquellas menos relevantes o carentes de significación estadística. Para ello hemos optado por el empleo de la prueba de Chi-cuadrado y el análisis de las tablas de contingencia, las más aptas en el marco del análisis de las variables cualitativas contempladas en nuestro estudio. 
Internet, redes sociales y adolescencia: un estudio en centros de Educación Secundaria de la región de Murcia

\section{RESULTADOS}

A continuación se realiza una exposición general de los resultados obtenidos. A través de un análisis descriptivo e inferencial.

\section{- Datos de identificación}

De los 119 participantes el género de la muestra es el siguiente: el $52.1 \%$ son chicas y el $47.9 \%$ chicos. En relación con su edad en el gráfico siguiente se observa que mayoritariamente son alumnos de 13 años, seguido de alumnos de 14 y 15 años.
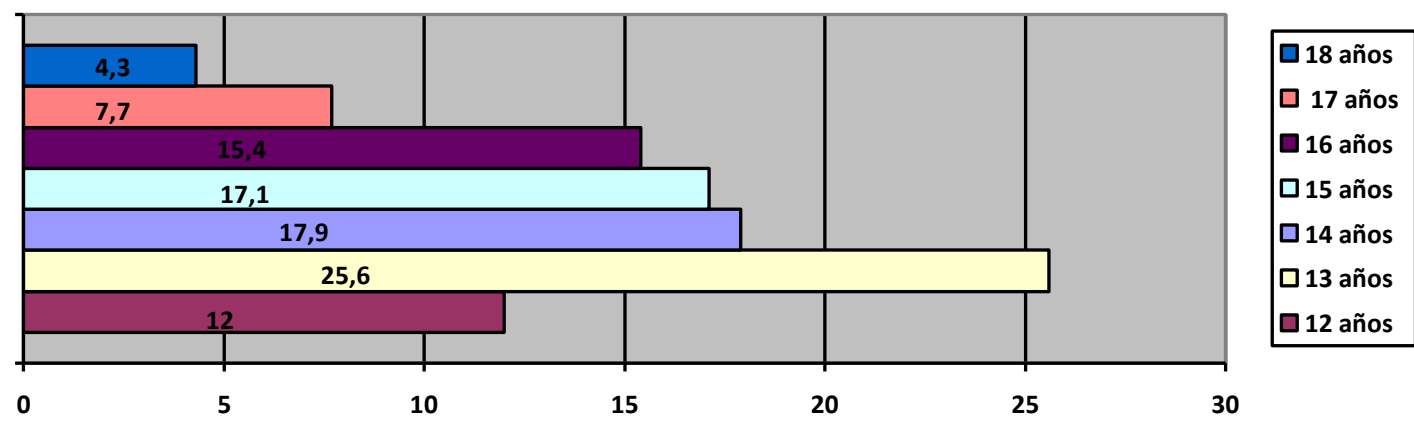

Gráfica n ${ }^{\circ} 1$. Edad de los participantes

La nacionalidad de los adolecentes participantes en este estudio queda recogida en la siguiente tabla donde se observa que el $80,7 \%$ son españoles, seguido del $5 \%$ de Ecuador y el $3.4 \%$ de Marruecos y Colombia, respectivamente.

\begin{tabular}{ll} 
España & 80,7 \\
\hline Marruecos & 3,4 \\
\hline Brasil & 0,8 \\
Bolivia & 0,8 \\
\hline Ecuador & 5,0 \\
Ucrania & 1,7 \\
Republica Dominicana & 0,8 \\
Colombia & 3,4 \\
Chile & 0,8 \\
\hline Azerbaiyán & 0,8 \\
\hline
\end{tabular}

Tabla $n^{\circ} 1$. Nacionalidad de la muestra

Con relación a la nacionalidad de los padres de la muestra, el $75.6 \%$ con autóctonos y el $24.4 \%$ extranjeros, de los cuales las 
nacionalidades con mayor frecuencia son Ecuador (6,7\%), Marruecos (4.2\%) y Colombia (3.4\%).

Si analizamos la procedencia de las madres de los participantes, el $76.5 \%$ son autóctonas frente al $23,5 \%$ que son de origen extranjero, siendo las nacionalidades más frecuentes Ecuador (6,7\%), Marruecos (5\%) y Colombia (3.4\%), al igual que ocurría con el grupo de los padres y de los jóvenes objeto de estudio. La información recogida en la tabla siguiente nos indica que los participantes que han implementado este cuestionario principalmente son de $1^{\circ}$ de la ESO, seguido de los de $3^{\circ}$ de la ESO, tal y como se muestra en el gráfico siguiente:

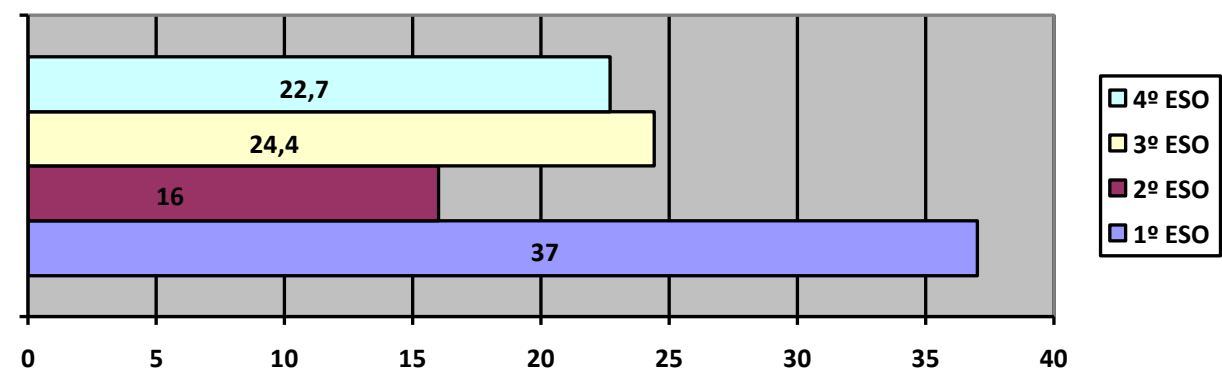

Gráfica n². Nivel de estudios

A continuación vamos a analizar los ítems de la dimensión "Tu tiempo libre" con el fin de exponer aquellos aspectos generales respecto de la influencia y el riesgo de las redes sociales en la vida diaria de los adolescentes de la muestra; así como el control que ejerce las familias sobre ese uso según los jóvenes de entre 13 al 18 años.

Si recibes una invitación en tu red social de un adulto desconocido, ìlo aceptas rápidamente como amigo?

Un $80.7 \%$ nunca acepta la invitación de un desconocido como amigo en las redes sociales, un $1.7 \%$ afirman si aceptarlo.

\begin{tabular}{lc}
\hline Nunca & $\mathbf{8 0 . 7 \%}$ \\
Casi nunca & $10.9 \%$ \\
Algunas veces & $3.4 \%$ \\
Casi siempre & $3.4 \%$ \\
Siempre & $1.7 \%$ \\
\hline
\end{tabular}

La $x^{2}(4)=22.30, p<0.000$, arroja un valor de significación que indica que existe una asociación estadísticamente significativa entre aceptar una invitación de un desconocido en las redes sociales y el origen (autóctono y extranjero) de los participantes. En concreto, un 
Internet, redes sociales y adolescencia: un estudio en centros de Educación Secundaria de la región de Murcia

$3.6 \%$ de los jóvenes extranjeros opinan que siempre aceptan invitaciones de personas desconocidas en las redes sociales.

- Las redes sociales ise pueden considerar un lugar seguro para los menores como yo?

En esta cuestión las opiniones son variadas casi un $60 \%$ lo consideran nunca o casi nunca un lugar seguro, un poco más de un cuarto $(26.9 \%)$ lo consideran algunas veces, siendo el $15 \%$ los que creen que es un lugar seguro.
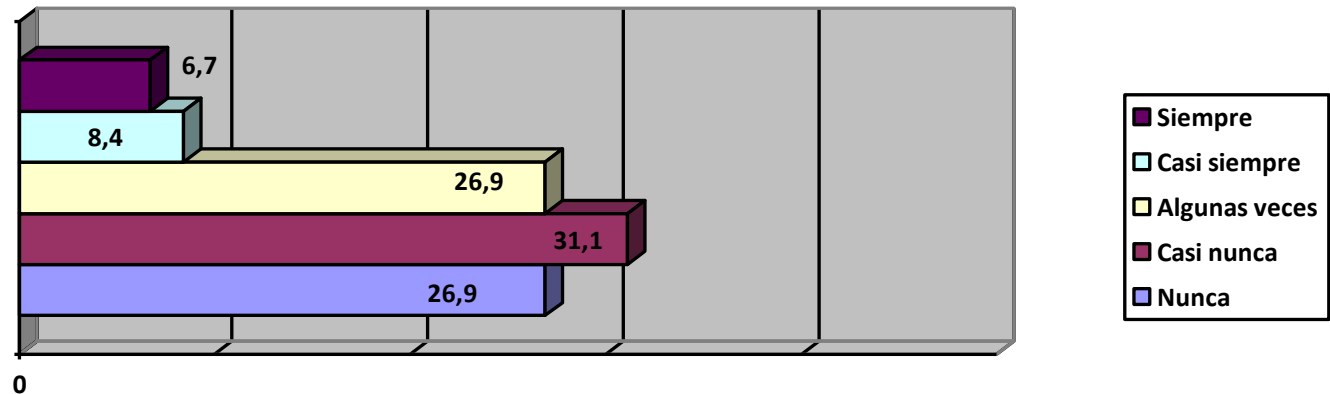

Gráfica n³. Redes sociales como lugar seguro

Analizando los ítems redes sociales como lugar seguro y aceptar a un desconocido adulto en las redes sociales como amigo, hemos comprobado que hay relación entre ambas variables. La $x^{2}(16)=42,64$ $\mathrm{p}<0.000$, arroja un valor de significación, esto es, existe una asociación estadísticamente significativa entre las redes sociales como lugar seguro y la aceptación de una invitación de un desconocido. Un 6.7\% de los jóvenes que piensan que las redes son un lugar seguro, son los que casi siempre o algunas veces (6.8\%) aceptan invitaciones de desconocidos adultos en la redes sociales.

- ¿Has contactado con personas a través de Internet sin conocerlas de nada?

Casi un $60 \%$ de los participantes opinan que nunca han contactado con desconocidos, aunque $16.8 \%$ lo han hecho algunas veces, además un $8.4 \%$ siempre o casi siempre reconocen haber contactado sin tener conocimiento de esas personas. 

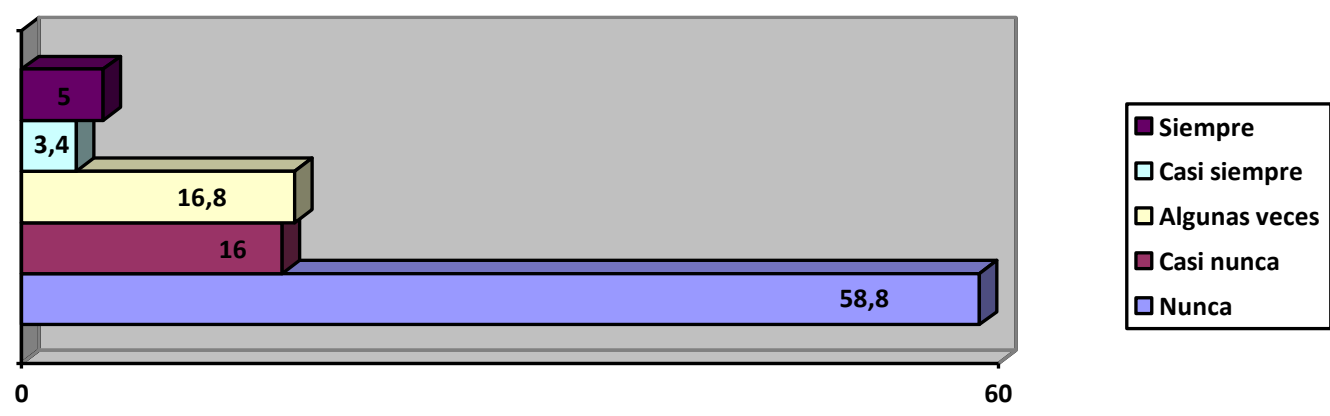

Gráfica n4. Contacto con desconocidos en la red

Así comprobamos que la $x^{2}(4)=19.05, p<0.001$, que indica que existe una asociación estadísticamente significativa entre contactar con desconocidos en Internet y el origen (autóctono y extranjero) de los participantes. Un $4.5 \%$ de los jóvenes extranjeros opinan que siempre contactan con desconocidos en la red frente al $0.8 \%$ de jóvenes autóctonos.

Cuando se cruza esta variable con el curso, la $x^{2}(12)=34.09, p<$ 0.000 , arroja un valor de significación entre contactar con desconocidos en Internet y el nivel de escolarización de los participantes (dentro de la Educación Secundaria Obligatoria, ESO). Un $4.2 \%$ de los jóvenes escolarizados en $2^{\circ}$ ESO opinan que siempre contactan con desconocidos en la red frente al $0.8 \%$ de $1^{\circ}$ ESO, ya que los alumnos de $3^{\circ}$ y $4^{\circ}$ de la ESO, no han marcado esa opción de respuesta.

Por otro lado también encontramos relación entre haber contactado con desconocidos y aceptar una invitación entre desconocidos, la $x^{2}(16)=95.542, p<0.000$. Un $1.7 \%$ de los jóvenes que opinan que siempre contactan con desconocidos en la red son los que aceptan invitaciones de desconocidos frente otro $1.7 \%$ que dicen que siempre contactan con desconocidos pero solo, algunas veces, aceptan su invitación en las redes sociales.

Los adolescentes consideran que Internet es un lugar seguro y por tanto contactan con desconocidos, puesto que hemos comprobado que existe una asociación estadísticamente significativa entre ambas variables $\left(x^{2}(16)=39.366, p<0.001\right)$. Un $4.2 \%$ de los jóvenes opinan que siempre o casi siempre contactan con desconocidos en la red siendo éstos los que consideran la red como un lugar seguro.

Valores muy parecidos obtenemos al relacionar este ítem con el de si ellos consideran Internet un lugar seguro para menores como él. La $x^{2}(16)=39.336, p<0.001$, nos indica que existe una asociación estadísticamente significativa entre contactar con desconocidos en 
Internet, redes sociales y adolescencia: un estudio en centros de Educación Secundaria de la región de Murcia

Internet y el hecho de considerar Internet un lugar seguro para menores como él. El $4.2 \%$ de los jóvenes que opinan que siempre o casi siempre contactan con desconocidos son los que consideran, también, que Internet es un lugar seguro para menores como ellos.

- Siempre "subo" mis fotos en mi red social para que cualquier persona, aunque no lo conozca, pueden verlas sin problemas.

Casi un $90 \%$ afirman que nunca o casi nunca cuelgan las fotos para todo el público, un $6.7 \%$ siempre las cuelga para que pueda acceder cualquier persona.

\begin{tabular}{lc}
\hline Nunca & $60.5 \%$ \\
Casi nunca & $27.7 \%$ \\
Algunas veces & $2.5 \%$ \\
Casi siempre & $2.5 \%$ \\
Siempre & $6.7 \%$ \\
\hline
\end{tabular}

Comprobamos que existe una asociación estadísticamente significativa entre "subir" las fotos públicamente en Internet y el origen (autóctono y extranjero) de los participantes $\left(x^{2}(4)=10.06, p<0.05\right)$. Un $54 \%$ de los jóvenes autóctonos opinan que nunca "suben" sus fotos públicamente en las redes sociales frente al $6.7 \%$ de jóvenes extranjeros. Por otro lado, hemos relacionado este ítem con aceptar la invitación de un desconocido adulto en las redes sociales, y los resultados que nos hemos encontrado son, también, estadísticamente significativos $(x 2$ $(16)=89.90, p<0.00)$. Un $4.2 \%$ de los jóvenes que siempre o casi siempre "suben" sus fotos públicamente en las redes sociales son los que siempre o casi siempre aceptan una invitación de desconocidos frente al 55.5\% de jóvenes que nunca "suben" fotos ni aceptan invitaciones de desconocidos

También hemos encontrado una asociación estadísticamente significativa entre los adolescentes que contactan con desconocidos y los que "suben" sus fotos a Internet $\left(x^{2}(16)=52.876, p<0.000\right)$ - Así, el $1.7 \%$ de los jóvenes opinan que, siempre, contactan con desconocidos en la red son los que, además, "suben" sus fotos a Internet frente al $45.5 \%$ que nunca realizan ninguna de estas dos acciones. Internet?

$$
\text { ¿ ¿Has tenido o intentado tener citas "a ciegas" a través de }
$$


Los adolescentes encuestados dicen que nunca $(87,4 \%)$, han tenido o han intentado tener una "cita a ciegas" a través de Internet, un $7.6 \%$ opinan que casi nunca, un $3,4 \%$ algunas veces y tan solo en $0.8 \%$ en casi siempre y siempre.

\begin{tabular}{lc}
\hline Nunca & $\mathbf{8 7 . 4 \%}$ \\
Casi nunca & $7.6 \%$ \\
Algunas veces & $3.4 \%$ \\
Casi siempre & $0.8 \%$ \\
Siempre & $0.8 \%$ \\
\hline
\end{tabular}

Tabla $n^{\circ} 4$. Cita con desconocidos

Los datos obtenidos nos indican que existe una asociación estadísticamente significativa entre tener citas o contactar con desconocidos a través de Internet y "subir" las fotos públicamente $(x 2$ $(16)=64.637, p<0.000$ ). Comprobamos que un $1.6 \%$ de los jóvenes opinan que siempre o casi siempre han tenido "citas a ciegas" en la red y estos mismos afirman que cuelgan sus fotos para que cualquier persona pueda tener acceso a ellas.

Estos jóvenes suelen tener citas a ciegas con desconocidos puesto que aceptan invitaciones de adultos desconocidos en la red $\left(x^{2}(16)=\right.$ 155.86, $\mathrm{p}<0.000$ ), Los datos arrojan que el $1.6 \%$ de los jóvenes opinan que siempre o casi siempre contactan con desconocidos en la red y estos mismos son los que suelen tener citas a ciegas con desconocidos en la red.

Por último, también, hemos encontrado que hay relación entre tener citas a través de Internet y contactar con personas sin conocerlas de nada. La $x^{2}(16)=62.60, p<0.000$, nos aporta que existe una asociación estadísticamente significativa entre tener citas a ciegas con desconocidos en Internet y haber contactado con personas a través de Internet sin conocerlas. Un $1.6 \%$ de los jóvenes opinan que siempre 0 casi siempre tiene citas a ciegas son los mismo que han contactado con personas desconocidas en Internet; frente a un $2.5 \%$ de los que afirman que, algunas veces, tiene citas a ciegas y contactan con desconocidos en Internet.

\section{- Me he sentido acosado a través de Internet}

Un $90.8 \%$ de los adolescentes opinan que nunca se han sentido acosados en Internet, un 5.9\% afirman que casi nunca y tan solo un $3.4 \%$ señalan que algunas veces. 
Internet, redes sociales y adolescencia: un estudio en centros de Educación Secundaria de la región de Murcia

Hemos pretendido valorar si existía alguna relación entre esta variable, es decir, si se habían sentido acosados en Internet y, por otro lado, si habían aceptado la invitación de un desconocido adulto a través de las redes sociales. Los datos obtenidos $\left(\chi^{2}(8)=34.969 \mathrm{p}<\right.$ 0.000 ) nos indican que existe era relación. Así, un $3.4 \%$ de los jóvenes que afirman que algunas veces se han sentido acosados, son los que también en algunas ocasiones (3.4\%) aceptan invitaciones de desconocidos adultos en la redes sociales.

Asimismo hay relación entre tener "citas a ciegas" en la red con haberse sentido acosado, $\left(x^{2}(8)=31.105, p<0.000\right)$. Comprobamos que el $1.7 \%$ de los jóvenes que, algunas veces, han tenido "citas a ciegas" en la red son los que también, algunas veces, se han sentido acosados.

- Si te has sentido acosado en Internet, żse lo has comunicado a tus padres?

Un $65 \%$ de los encuestados afirman que nunca se lo han comunicado a sus padres cuando han sido acosados en Internet, frente al 25.2\% que señalan que siempre que les ha sucedido esta situación de bullying se lo han dicho a sus padres.

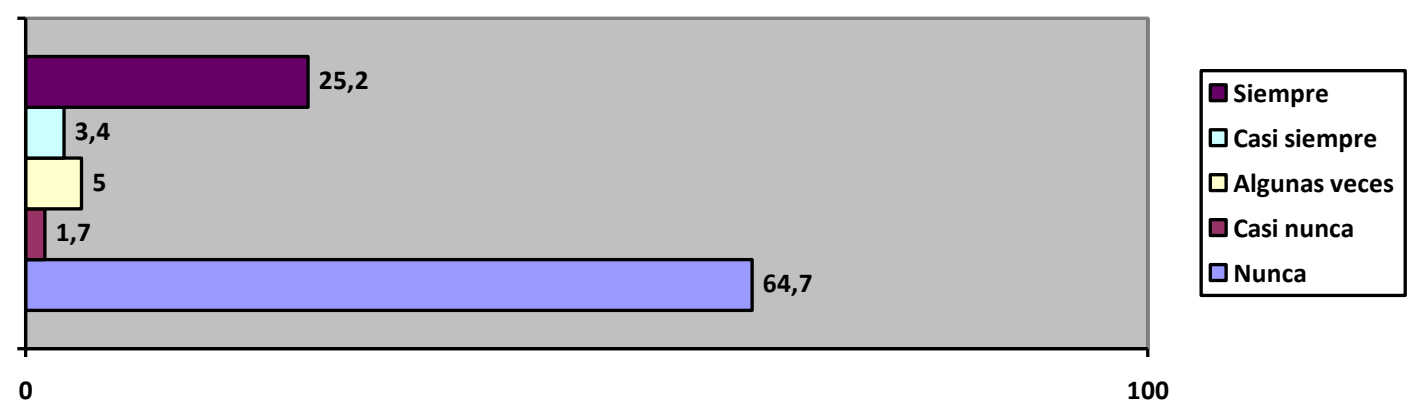

Gráfica n5. Comunicación tras el acoso

- Cuando navegas por Internet, ¿̇tu familia o un adulto vigilan lo que haces?

Más de la mitad de los participantes afirman que nunca son vigilados por ningún familiar cuando están en Internet. Tan solo un $12.6 \%$ opinan que siempre o casi siempre son vigilados.

\begin{tabular}{lc}
\hline Nunca & $54.6 \%$ \\
\hline Casi nunca & $19.3 \%$ \\
Algunas veces & $13.4 \%$ \\
Casi siempre & $7.6 \%$ \\
\hline
\end{tabular}




\section{Siempre}

$5 \%$

Tabla $n^{\circ} 5$. Vigilan los familiares el acceso a Internet

- Mis padres me dicen a qué páginas webs puedo entrar y a qué redes sociales me puedo dar de alta

Un $65.6 \%$ de los participantes opinan que casi nunca o nunca sus padres saben donde acceden a Internet y donde se dan de alta en las redes sociales. Un 11,8\% consideran que siempre son sus padres los que les ofrecen el acceso a Internet y a las redes sociales.
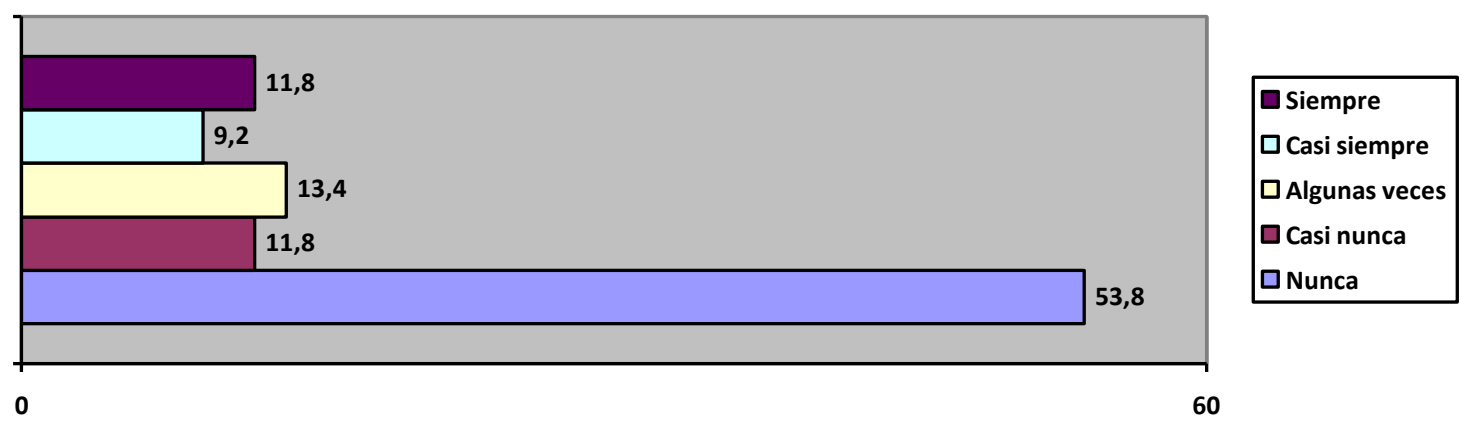

Gráfica 6. Control familiar del acceso a páginas web y a redes sociales

Hemos relacionado los dos ítems anteriores, y una vez analizado, hemos comprobado que hay una asociación estadísticamente significativa entre ambos ítems. Los resultados obtenidos $\left(\varkappa^{2}(16)=55.13\right.$, $p<0.000$ ) nos indican que aquellos adolescentes que son vigilados $(5 \%)$ cuando acceden a Internet son los que sus padres controlan su acceso $(11,8 \%)$; en contraposición al $73,9 \%$ de los jóvenes que nunca o casi nunca son vigilados, y que, tampoco, son regulados o controlados por sus padres.

\section{DISCUSIÓN Y CONCLUSIONES}

Los resultados de esta investigación nos muestran que en relación al primer objetivo: "Conocer la influencia y el riesgo de las redes sociales en las relaciones de los adolescentes", los datos nos dicen que aunque el $80,7 \%$ de los participantes no aceptan la invitación de un desconocido en las redes sociales, los jóvenes de nacionalidad extranjera suelen aceptar más invitaciones de personas desconocidas que los españoles.

Según los jóvenes participantes en esta muestra, un $60 \%$ no consideran las redes sociales como un lugar seguro, un $6.7 \%$ de los jóvenes que piensan que las redes son un lugar seguro, son los que casi siempre o algunas veces (6.8\%) los que aceptan invitaciones de 
Internet, redes sociales y adolescencia: un estudio en centros de Educación Secundaria de la región de Murcia

desconocidos adultos en redes sociales. Aunque los que no consideran Internet un lugar seguro sí que suelen aceptar invitaciones a desconocidos por lo que de nuevo vuelve a aparecer la inseguridad en la red, ya que se demuestra que los adolescentes no advierten el peligro del uso de las redes sociales.

Casi un $75 \%$ de los jóvenes opinan que nunca o casi nunca han contactado con personas a través de las redes sociales sin conocerlas de nada pero todavía hay un porcentaje de alumnos que sí lo hace. Según la nacionalidad del alumnado, un $4.5 \%$ de los jóvenes extranjeros opinan que siempre contactan con desconocidos en la red frente al $0.8 \%$ de jóvenes autóctonos. Atendiendo al nivel educativo, un $4.2 \%$ de los jóvenes escolarizados en $2^{\circ} \mathrm{ESO}$ opinan que siempre contactan con desconocidos en la red frente al $0.8 \%$ de $1^{\circ} \mathrm{ESO}$, ya que los alumnos de $3^{\circ}$ y $4^{\circ}$ de la ESO, no han marcado esa opción de respuesta. Un $1.7 \%$ de los jóvenes opinan que siempre contactan con desconocidos en la red y son los que aceptan estas invitaciones frente otro $1.7 \%$ que dicen que siempre contactan con desconocidos pero solo algunas veces aceptan su invitación en las redes sociales. Precisamente ese $4.2 \%$ de los jóvenes que opinan que siempre o casi siempre contactan con desconocidos en la red son los que consideran la red como un lugar seguro y también, para menores como ellos.

De los resultados extraídos podemos señalar que los jóvenes autóctonos son más cautelosos a la hora de usar las redes sociales que los jóvenes extranjeros, aspecto en el que coincidimos con el estudio realizado por Soriano-Ayala \& González-Jiménez (2014). Al mismo tiempo que también se destaca que los participantes de menor edad, escolarizados en los primeros cursos de la ESO, no consideran el uso de las redes sociales tan peligrosos en su vida diaria como los escolarizados en cursos superiores.

La mayoría nunca o casi nunca "sube" fotos a Internet públicamente, siendo los alumnos extranjeros $(6.7 \%)$ más propicios para realizar esta acción frente al $54 \%$ de los jóvenes autóctonos que afirman que nunca "suben" sus fotos públicamente en las redes sociales.

Un $4.2 \%$ de los jóvenes que siempre o casi siempre "suben" sus fotos públicamente en las redes sociales son los que siempre o casi siempre aceptan una invitación de desconocidos frente al $55.5 \%$ de jóvenes que nunca "suben" fotos ni aceptan invitaciones de desconocidos. También un $1.7 \%$ de los jóvenes opinan que siempre contactan con desconocidos en la red son los que "suben" sus fotos a Internet frente al $45.5 \%$ que nunca realizan ninguna de estas dos acciones. 
Un 95\% nunca o casi nunca han tenido una "cita a ciegas", a través de las redes sociales. Un $1.6 \%$ de los jóvenes opinan que siempre o casi siempre han tenido "citas a ciegas" en la red y estos mismos afirman que "suben" sus fotos para que cualquier persona pueda tener acceso a ellas.

Casi un $90 \%$ de los jóvenes opinan que nunca o casi nunca se han sentido acosados a través de la red. Un $3.4 \%$ de los jóvenes que afirman que algunas veces se han sentido acosados, son los que también en algunas ocasiones (3.4\%) aceptan invitaciones de desconocidos adultos en la redes sociales. También hay relación entre tener "citas a ciegas" en la red con haberse sentido acosado, un $1.7 \%$ de los jóvenes que algunas veces han tenido "citas a ciegas" en la red son los que también algunas veces se han sentido acosados. Teniendo en cuenta los datos aportados podemos comprobar que todavía, en la actualidad, hay un pequeño grupo de alumnos que no valoran el riesgo que conlleva el uso desprotegido de las redes sociales, pues "suben" fotos públicamente y aceptan una invitación de desconocidos. Estos además tienen una cita a ciegas y han sido acosados en alguna ocasión. Si tenemos en cuenta la nacionalidad comprobamos que están más desprotegidos los alumnos de origen extranjero que los autóctonos.

En relación con el segundo objetivo: "Valorar el papel de las familias sobre el uso de sus hijos de Internet", siempre desde la percepción que hacen sus hijos que son los alumnos de los centros de Educación Secundaria objeto de estudio. Por sus comentarios los padres no vigilan donde aceden sus hijos en Internet, lo que nos indica que no existe un control sobre el uso de las redes sociales de los jóvenes actualmente.

Un $65 \%$ de los encuestados afirman que nunca se lo han comunicado a sus padres cuando han sido acosados en Internet, frente al 25.2\% que señalan que siempre que les ha sucedido esta situación de bullying se lo han dicho a sus padres.

Cuando relacionamos el control de los padres al acceder a Internet y la orientación o regulación que ejercen ante el uso de las páginas web y redes sociales, encontramos que el $73,9 \%$ de los jóvenes nunca o casi nunca son vigilados, y por tanto, tampoco regulados 0 controlados, y que aquellos padres que regulan el acceso son los que, además, orientan a sus hijos a la hora de utilizar determinadas páginas web y redes sociales. 
Internet, redes sociales y adolescencia: un estudio en centros de Educación Secundaria de la región de Murcia

Al valorar la regulación familiar sobre el acceso a páginas web y redes sociales, un $53.8 \%$ de adolescentes señalan que su familia no los controlan a la hora de navegar por Internet, además un $65.6 \%$ de los participantes opinan que casi nunca o nunca sus padres les dicen o les orientan sobre qué páginas web pueden acceder y a qué redes sociales pueden darse de alta.

Los jóvenes acceden a todas las redes sociales y a Internet sin que sus padres comprueben la seguridad de sus hijos en la red. Por lo que es necesario, que desde el ámbito educativo se establezcan programas para ayudar a los jóvenes a ser críticos con la información, el uso y consumo de Internet y las redes sociales. Y al mismo tiempo, emplear diferentes formas de informar a los padres sobre la seguridad en la red y los sitios que, usualmente, sus hijos visitan.

Para concluir señalar, que la mayoría del alumnado considera que Internet es un lugar seguro y que nunca han sido acosados a través de las redes sociales, al mismo tiempo que toman medidas de seguridad al "subir" imágenes, puesto que un $60 \%$ nunca lo hacen públicamente, ni tampoco contactan con desconocidos o han tenido una cita con personas que no conocen. Aunque hay un grupo muy reducido (5\%) que siempre "suben" fotos a Internet y al mismo tiempo son los que contactan con desconocidos, por lo que ellos no son conscientes el riesgo que conlleva el acceso a Internet cuando no protegemos nuestra privacidad. Los jóvenes consideran que contactar con desconocidos no conlleva ningún peligro para ellos, puesto que aquellos adolescentes que siempre o casi siempre contactan con desconocidos son los que "suben" fotos, aceptan invitaciones de adultos desconocidos en la red, consideran Internet un lugar seguro para ello mismos así como para menores de edad, los que nos lleva a concluir que los jóvenes no conocen de los peligros del mal uso de las redes, puesto que no son críticos con un uso razonable del mismo, por tanto, se debería formar a los jóvenes sobre los riesgos que conlleva el acceso y el uso de las redes en la actualidad. De igual forma, "Ias citas a ciegas" con desconocidos en la red es un fenómeno que los adolescentes participantes en la muestra realizan frecuentemente, puesto que ellos mismos afirman que mantienen relaciones con desconocidos, cuelgan fotos públicamente, sentirse acosado y contactan con personas desconocidas a través de la red. Los jóvenes no ven un uso inadecuado de las redes sociales y piensan que la influencia en sus vidas no les perjudica.

Si nuestros jóvenes van a crecer en un mundo digital, necesitamos una educación específica que favorezca su uso y elimine aspectos que son peligrosos para nuestros menores (González-Jiménez, 2013). 
Desafortunadamente conductas como sexting, ciberbullying y acoso son prácticas que ocurren todos los días en las redes sociales. Como afirman Tejedor \& Pulido (2012) el acoso entre iguales mediado por las interacciones on-line, genera un impacto muy negativo en su desarrollo emocional; depresiones, baja autoestima, y en el último extremo el suicidio, además señalan la importancia de promover una formación crítica y prevención no solo limitada a los y las menores, sino ampliada a toda la comunidad. Estimamos que ante la influencia que los medios digitales están protagonizando en los jóvenes, la educación no puede mirar para otro lado.

\section{REFERENCIAS BIBLIOGRÁFICAS}

Ballesta, J., Lozano, J \& Cerezo, M. (2014). El uso y consumo de TIC en el alumnado autóctono y extranjero de Educación Secundaria Obligatoria de la Región de Murcia. Revista de Educación a Distancia, 41. Número especial "Interculturalidad en el nuevo paradigma educativo". Recuperado 20/10/2014 de http://www.um.es/ead/Red_U/m4/

Becoña, E. (2006). Adicción a nuevas tecnologías. Vigo: Nova Galicia Edicións

Bernal, C. \& Angulo, F. (2012). Interacciones de los jóvenes andaluces en las redes sociales. Comunicar, 20 (40), 25-30.

Bisquerra, R. (1989). Métodos de investigación educativa. Guía educativa. Barcelona: CEAC.

Bisquerra, R. (Coord.) (2012). Metodología de la Investigación Educativa ( $3^{a}$ edición). Madrid: La Muralla.

Bringué, X \& Sádaba, C. (2009). La Generación interactiva en España. Niños y adolescentes ante las pantallas. Madrid: Ariel-Fundación Telefónica.

Buckingham, D. (2007). Digital Media Literacies: Rethinking media education in the age of the Internet. Research in Comparative and International Education, 2(1), 43-55.

DOI: $\mathrm{http://dx.doi.org/10.2304/rcie.2007.2.1.43}$

Buckingham, D. (2008). Digital culture, Media education and the Place of Schooling. Recuperado 10/09/2011 de www.childrenyouthandmediacentre.co.uk

Cohen, J. (1992). A power primer. Psychological bulletin, 112 (1), 155.

DOI: http://dx.doi.org/10.1037/0033-2909.112.1.155

Cohen, J. (2013). Statistical power analysis for the behavioral sciences. New York: Academic press.

Colás, P., González, T. \& de Pablos, J. (2013). Juventud y redes sociales: motivaciones y usos preferentes. Comunicar, 20(40), 15-23.

Durán, M. \& Guerra, J. M. (2015). Usos y tendencias adictivas de una muestra de estudiantes universitarios españoles a la red social 
Internet, redes sociales y adolescencia: un estudio en centros de Educación Secundaria de la región de Murcia

Tuenti: La actitud positiva hacia la presencia de la madre en la red como factor protector. Anales de Ppsicología, 31 (1), 260-267. DOI: http://dx.doi.org/10.6018/analesps.31.1.158301

Fundación Telefónica (2013) Guía Mobile Learning. Recuperado de http://www.fundacion.telefonica.com/es/index.htm.

González-Jiménez, A.J. (2013) Aprendizaje-Servicio. Educar en las competencias emocionales mediante el Mobile Learning. En Soriano-Ayala, E. (Ed.) Interculturalidad y Neocomunicación (161181). Madrid: La Murallla.

Livingstone, S. (2008). Taking risky opportunities in youthful content creation: teenagers'use of social networking sites for intimacy, privacy and self-expression. New Media and Society, 10, 393-411.

DOI: http://dx.doi.org/10.1177/1461444808089415

Livingstone, S. y Haddon, L. (2011). EU Kids Online III. A new project. London: LSE, EU Kids Online. Recuperado de http://www2.Ise.ac.uk/media@lse/research/EUKidsOnline/Home.as $\underline{\mathrm{px}}$

McCarty, C., Prawitz, A. D., Derscheid, L. E. y Montgomery, B. (2011). Perceived safety and teen risk taking in online chat sites. Cyberpsychology, Behavior, and Social Networking, 14, 174-184. DOI: http://dx.doi.org/10.1089/cyber.2010.0050

O'Keeffe, G. S. \& Clarke-Pearson, K. (2011). Clinical Report - The Impact of Social Media on Children, Adolescents, and Families. Pediatrics, 127, 800-804. DOI: http://dx.doi.org/10.1542/peds.2011-0054

Orange \& Observatorio del Instituto Nacional de las Tecnologías de Información (2010) Estudio sobre la seguridad y privacidad en el uso de los servicios móviles españoles. Recuperado de http://www.inteco.es

Rial. A., Gómez, P., Braña, T. \& Varela, J. (2014). Actitudes, percepciones y uso de Internet y las redes sociales entre los adolescentes de la comunidad gallega. Anales de Psicología, 30 (2), 642-655.

DOI: http://dx.doi.org/10.6018/analesps.30.2.159111

Romero, F. \& Diez, F. (2013) El Observatorio de las redes sociales. Índice Revista de Estadística y Sociedad, 55, 24-26.

Rubio, A. (Dir.) (2009). Adolescentes y jóvenes en la red: factores de oportunidad. Madrid: Injuve.

Sánchez, A \& Fernández, Ma P., (2010). Informe Generación 2.0: Hábitos de los adolescentes en el uso de las redes sociales. Estudio comparativo entre Comunidades Autónomas. Madrid: Universidad Camilo José Cela.

Soriano-Ayala, E. \& González-Jiménez, A. (2014). Spanish and Moroccan youths on social networks: A quantitative study in Spain. Procedia Social and Behavioral Sciences 132, $32-36$

DOI: http://dx.doi.org/10.1016/i.sbspro.2014.04.274 
Tejedor, S \& Pulido, C (2012). Retos y riesgos del uso de internet por parte de los menores ¿Cómo empoderarlos? Comunicar, 20 (39), 65-72.

Unicef (2012) La seguridad de los niños en línea: Retos y estrategias mundiales. Florencia: Centro de Investigación Innocenti de Unicef

\section{Sobre los autores:}

\section{F. Javier Ballesta Pagán}

pagan@um.es

Universidad de Murcia

Profesor Titular de Tecnología Educativa en el Departamento de Didáctica y Organización Escolar de la Facultad de Educación de la Universidad de Murcia. Su actividad investigadora se centra en el ámbito de la comunicación y la innovación educativa, la Educación Mediática y la formación para el uso de las TIC. Ha coordinado proyectos sobre El consumo de medios digitales y El uso y la interacción de las Tecnologías de la Información y la Comunicación (TIC) en el alumnado. Investigador del grupo (E073-05) Comunicación, Innovación Educativa y Atención a la Diversidad (EDUCODI) y Editor de la revista Educatio Siglo XXI de la Universidad de Murcia.

\section{Josefina Lozano Martínez}

lozanoma@um.es

Universidad de Murcia

Profesora Titular de la Universidad de Murcia en la Facultad de Educación dentro del Departamento de Didáctica y Organización Escolar. Pertenece al grupo de investigación (E073-05) Comunicación, Innovación Educativa y Atención a la Diversidad" (EDUCODI), de la Universidad de Murcia, a través del cual, ha dirigido proyectos de investigación subvencionados, centrados en la educación intercultural y la atención al alumnado con necesidad específica de apoyo educativo. Entre sus publicaciones destacamos: "Proyecto curricular para la diversidad: psicomotricidad y lectoescritura", "Educar en la diversidad", "El euro para todos", "Adaptaciones curriculares para la diversidad", Colección de Materiales Didácticos "Aprendiendo el lenguaje con Nora" y "Diccionario Pictografíado de Nora", "Aprende con Zapo: Propuestas didácticas para el aprendizaje de habilidades emocionales y sociales. Respuesta educativa a las personas con trastorno del espectro autista", "Plan de Atención a la Diversidad". 
Internet, redes sociales y adolescencia: un estudio en centros de Educación Secundaria de la región de Murcia

\section{$M^{a}$ Carmen Cerezo Máiquez}

mcarmen.cerezo@um.es Universidad de Murcia

Diplomada en Magisterio, Licenciada en Pedagogía y Licenciada en Psicopedagogía. Profesora Asociada en el Departamento de Didáctica y Organización Escolar de la Facultad de Educación de la Universidad de Murcia. Ha realizado trabajos de investigación sobre la educación inclusiva, educación intercultural y la enseñanza del español como segunda lengua, teniendo algunas publicaciones y comunicaciones sobre esta temática. Sus líneas de investigación actuales son la educación intercultural y la enseñanza del español como segunda lengua. Entre sus publicaciones: Colección de Materiales Didácticos "Aprendiendo el lenguaje con Nora", "Diccionario Pictografíado de Nora" y "Plan de Atención a la Diversidad". Investigador del grupo de Investigación (E073-05), Comunicación, Innovación educativa y Atención a la Diversidad (EDUCODI) de la Universidad de Murcia.

\section{Encarnación Soriano Ayala}

esoriano@ual.es

Universidad de Almería

Profesora Titular de Métodos de Investigación en el Departamento de Educación de la Universidad de Almería. Ha llevado a cabo investigaciones en el ámbito de la Educación Intercultural. Sus líneas de investigación más destacadas son: Identidad cultural, Mediación intercultural, Ciudadanía Intercultural, Interculturalidad y género y la Educación para la Salud Transcultural. Es responsable del grupo de investigación HUM665 Investigación y Evaluación en Educación Intercultural.

\section{Para citar este artículo:}

Ballesta, J. et al. (2015). Internet, redes sociales y adolescencia: un estudio en centros de educación secundaria de la región de Murcia. Revista Fuentes, 16, Junio, pp. 109-130. [Fecha de consulta: $\mathrm{dd} / \mathrm{mm} /$ aada]. http://www.revistafuentes.es/

D.O.I.: http://dx.doi.org/10.12795/revistafuentes.2015.i16.05 